\title{
Modeling of Electrocardiogram Signals Using Predefined Signature and Envelope Vector Sets
}

\author{
Hakan Gürkan, ${ }^{1}$ Ümit Güz, ${ }^{1,2}$ and B. Sıddık Yarman ${ }^{3,4}$ \\ ${ }^{1}$ Department of Electronics Engineering, Engineering Faculty, IŞIK University, Kumbaba Mevkii, 34980 Sile, Istanbul, Turkey \\ ${ }^{2}$ Speech Technology and Research (STAR) Laboratory, Information and Computing Sciences Division, \\ SRI International, 333 Ravenswood Avenue, Menlo Park, CA 94025, USA \\ ${ }^{3}$ Department of Electrical-Electronics Engineering, College of Engineering, Istanbul University, 34230 Avcllar, Istanbul, Turkey \\ ${ }^{4}$ Department of Physical Electronics, Graduate School of Science and Technology, Tokyo Institute of Technology, \\ Ookayama Campus, 2-12-1 Ookayama, Meguro-Ku 152-8552, Tokyo, Japan
}

Received 28 April 2006; Accepted 24 November 2006

Recommended by Maurice Cohen

\begin{abstract}
A novel method is proposed to model ECG signals by means of "predefined signature and envelope vector sets (PSEVS).” On a frame basis, an ECG signal is reconstructed by multiplying three model parameters, namely, predefined signature vector (PSV) $R$ "predefined envelope vector $(\mathrm{PEV})_{K}$," and frame-scaling coefficient (FSC). All the PSVs and PEVs are labeled and stored in their respective sets to describe the signal in the reconstruction process. In this case, an ECG signal frame is modeled by means of the members of these sets labeled with indices $R$ and $K$ and the frame-scaling coefficient, in the least mean square sense. The proposed method is assessed through the use of percentage root-mean-square difference (PRD) and visual inspection measures. Assessment results reveal that the proposed method provides significant data compression ratio (CR) with low-level PRD values while preserving diagnostic information. This fact significantly reduces the bandwidth of communication in telediagnosis operations.
\end{abstract}

Copyright @ 2007 Hakan Gürkan et al. This is an open access article distributed under the Creative Commons Attribution License, which permits unrestricted use, distribution, and reproduction in any medium, provided the original work is properly cited.

\section{INTRODUCTION}

An electrocardiogram (ECG) signal which is a graphical display of the electrical activity of the heart is an essential biological signal for the monitoring and diagnosis of heart diseases. ECG signals which are recorded with digital equipment are most widely used in applications such as monitoring, cardiac diagnosis, event analysis, real-time transmission over telephone networks, patient databases, or long-term recording. The amount of ECG data grows depending upon sampling rate, sampling precision, number of lead, and recording time. Obviously, continuous generation of huge amount of ECG data requires high storage capacity and also wide transmission band for the remote monitoring activities. While retaining all clinically significant features including P-waves, QRS complexes, and T-waves, compression of the ECG signals is essential in the biomedical engineering [1-3].

Various methods have been developed for modeling and compression of ECG signals during the last thirty years $[2,3]$. These methods can be classified into the following three categories: direct time-domain techniques [2-8] attempt to reduce redundancy in the actual signal samples. Examples of this method include amplitude zone time epoch coding (AZTEC), the coordinate reduction time encoding system (CORTES), FAN and SAPA techniques, SAIES, mean-shape vector quantization method, gain-shape vector quantization method, and so forth. Transform-domain techniques [9-15] generate a coefficient sequence that reduces the amount of data needed to represent the original signal, and then inverse transformation is applied in order to reconstruct original signal with acceptable error. In this technique, discrete cosine transform (DCT), Karhunen-Loeve transform (KLT), singular value decomposition (SVD), wavelet transform (WT), and so forth have been employed for ECG compression and modeling. Parametric extraction methods [16] such as peakpeaking methods, linear prediction methods, neural network methods generate a set of parameters which is extracted from the original signal. These different modeling or compression methods yield different results, with regard to compression ratio and reconstruction error.

In the literature, [3] presented an ECG compressor which employs the zero-mean ECG segments and their associated 
mean values that are coded by vector and scalar quantization, respectively. In [5], an ECG signal compression technique using overlapped and linearly shifted codevectors is presented. Beat-based ECG compression method which exploits the redundancy among adjacent heartbeats and adjacent samples of the original ECG signals by using gain-shape vector quantization method is published in [6]. In [7], the ECG compression method is described for signal-dependent frames using matching pursuit algorithm. An algorithm based on approximate multiscale pattern matching, encoding segments of an input signal using expanded and contracted versions of patterns stored in a dictionary is proposed to compress ECG signals in [8]. ECG compression methods are proposed based on discrete cosine transform and singular value decomposition in $[9,12]$ and in $[10]$, respectively. Several ECG compression techniques based on wavelet transform are proposed in [11, 13-15]. An ECG compressor consists of beat codebook, short and long predictors, and an adaptive residual quantizer is presented in [16].

In our previous work $[17,18]$, speech signals were modeled by using predefined signature and envelope functions sets. In this work, the modeling method introduced in [18] is applied to represent ECG signals. Thus, in the reconstruction of ECG signal, while preserving diagnostic information; high compression ratios (CR) with acceptable percentage rootmean-square difference (PRD) levels are obtained.

In the following, first the proposed ECG modeling method is summarized. Then, formations of the predefined signature and envelope sets (PSEVS) are detailed (Section 2). In Section 3, an algorithm which generates the predefined signature and envelope sets and the reconstruction algorithm of the ECG signals are given. Section 4 is devoted to the test results where the performance of the proposed method is compared with those of conventional methods. Section 5 is spared for discussions and conclusion.

\section{A MATHEMATICAL METHOD TO MODEL ECG SIGNALS}

\subsection{Main statement}

For any time frame " $i$," the digitized ECG signal which is designated by vector $X_{i}$ can be expressed as

$$
X_{i} \cong C_{i} \alpha_{K} \varphi_{R},
$$

where $K \in\left\{1,2, \ldots, N_{E}\right\}, R \in\left\{1,2, \ldots, N_{S}\right\} ; K, R, N_{E}$, and, $N_{S}$ are integers. $C_{i}$ is a real constant, $\varphi_{R}^{T}=$ $\left[\varphi_{1}, \varphi_{2}, \varphi_{3}, \ldots, \varphi_{L F}\right]$ is a row vector. The vector $C_{i} \varphi_{R}$ carries almost maximum energy of $X_{i}$ in the LMS sense. In other words, $C_{i} \varphi_{R}$ is the best approximation of $X_{i}$ with one term that minimizes the sum of square error point by point, over the frame under consideration. The $\left(L_{F} \times L_{F}\right)$ diagonal ma$\operatorname{trix} \alpha_{K}=\operatorname{diag}\left\lfloor\begin{array}{lllll}\alpha_{i 1} & \alpha_{i 2} & \alpha_{i 3} & \cdots & \left.\alpha_{i L_{F}}\right\rfloor \text { acts as an envelope }\end{array}\right.$ term on the quantity $C_{i} \varphi_{R}$, which may satisfy the equality of $X_{i}=C_{i} \alpha_{K} \varphi_{R}$ or reduce the error defined on the difference $\left[X_{i}-C_{i} \alpha_{K} \varphi_{R}\right]$ in the least mean-square (LMS) sense. Thus, it matches the envelope of $C_{i} \varphi_{R}$ to the original ECG frame vector $X_{i}$. The integer $L_{F}$ designates the total number of elements in a frame " $i$."
Therefore, we introduce the following definitions.

Definition 1. The vector $\varphi_{R}$ is called the predefined signature vector (PSV) since it carries almost maximum energy of the ECG frame vector $X_{i}$ with a constant $C_{i}$.

Definition 2. The diagonal matrix $\alpha_{K}$ is called the predefined envelope matrix (PEM) since it matches the envelope of $C_{i} \varphi_{R}$ to the original ECG frame vector $X_{i}$.

Definition 3. The real constant $C_{i}$ is called the frame scaling coefficient (FSC).

\subsection{Verification of the main statement}

A discrete ECG signal $x(n)$ can be written as

$$
x(n)=\sum_{i=1}^{N} x_{i} \delta_{i}(n-i) .
$$

In this equation, $\delta_{i}(n)$ represents the unit sample; $x_{i}$ designates the amplitude of the sequence $x(n)$ of length $N . x(n)$ can also be given employing the vector/matrix notation,

$$
X^{T}=\left[\begin{array}{llll}
x(1) & x(2) & \cdots & x(N)
\end{array}\right]=\left[\begin{array}{llll}
x_{1} & x_{2} & \cdots & x_{N}
\end{array}\right] .
$$

In this representation, $X$ is called the main frame vector and it is divided into frames with equal lengths for example, 8, 16 , or 32 samples, and so forth. In this case, the frame matrix that is represented by $M_{F}$ is obtained by means of the frame vectors,

$$
M_{F}=\left[\begin{array}{llll}
X_{1} & X_{2} & \cdots & X_{N_{F}}
\end{array}\right]
$$

where

$$
X_{i}=\left[\begin{array}{llll}
x_{(i-1) L_{F}+1} & x_{(i-1) L_{F}+2} & \cdots & x_{i L_{F}}
\end{array}\right]^{T}, \quad i=1,2, \ldots, N_{F} .
$$

In this equation, $N_{F}=N / L_{F}$ designates the total number of frames in $X$. It can be shown that each frame vector $X_{i}$ can be spanned to a vector space formed by the orthonormal vectors $\left\{V_{k i} ; k=1,2,3, \ldots, L_{F}\right\}$, such that

$$
X_{i}=\sum_{k=1}^{L_{F}} c_{k} V_{k i}, \quad c_{k}=X_{i}^{T} V_{k i} .
$$

$V_{k i}$ are determined by minimizing the expected value of the error vector $\varepsilon=X_{i}-\sum_{k=1}^{L_{F}} c_{k} V_{k i}$ with respect to $V_{k i}$ in the LMS sense. Eventually, $V_{k i}$ are computed as the eigenvectors of the autocorrelation matrix $R_{i}$ of the frame sequence $X_{i}$ and it is given by

$$
R_{i}=\left[\begin{array}{ccccc}
r_{i}(1) & r_{i}(2) & r_{i}(3) & \cdots & r_{i}\left(L_{F}\right) \\
r_{i}(2) & r_{i}(1) & r_{i}(2) & \cdots & r_{i}\left(L_{F}-1\right) \\
r_{i}(3) & r_{i}(2) & r_{i}(1) & \cdots & r_{i}\left(L_{F}-2\right) \\
\vdots & \vdots & \vdots & \ddots & \vdots \\
r_{i}\left(L_{F}\right) & r_{i}\left(L_{F}-1\right) & r_{i}\left(L_{F}-2\right) & \cdots & r_{i}(1)
\end{array}\right]
$$



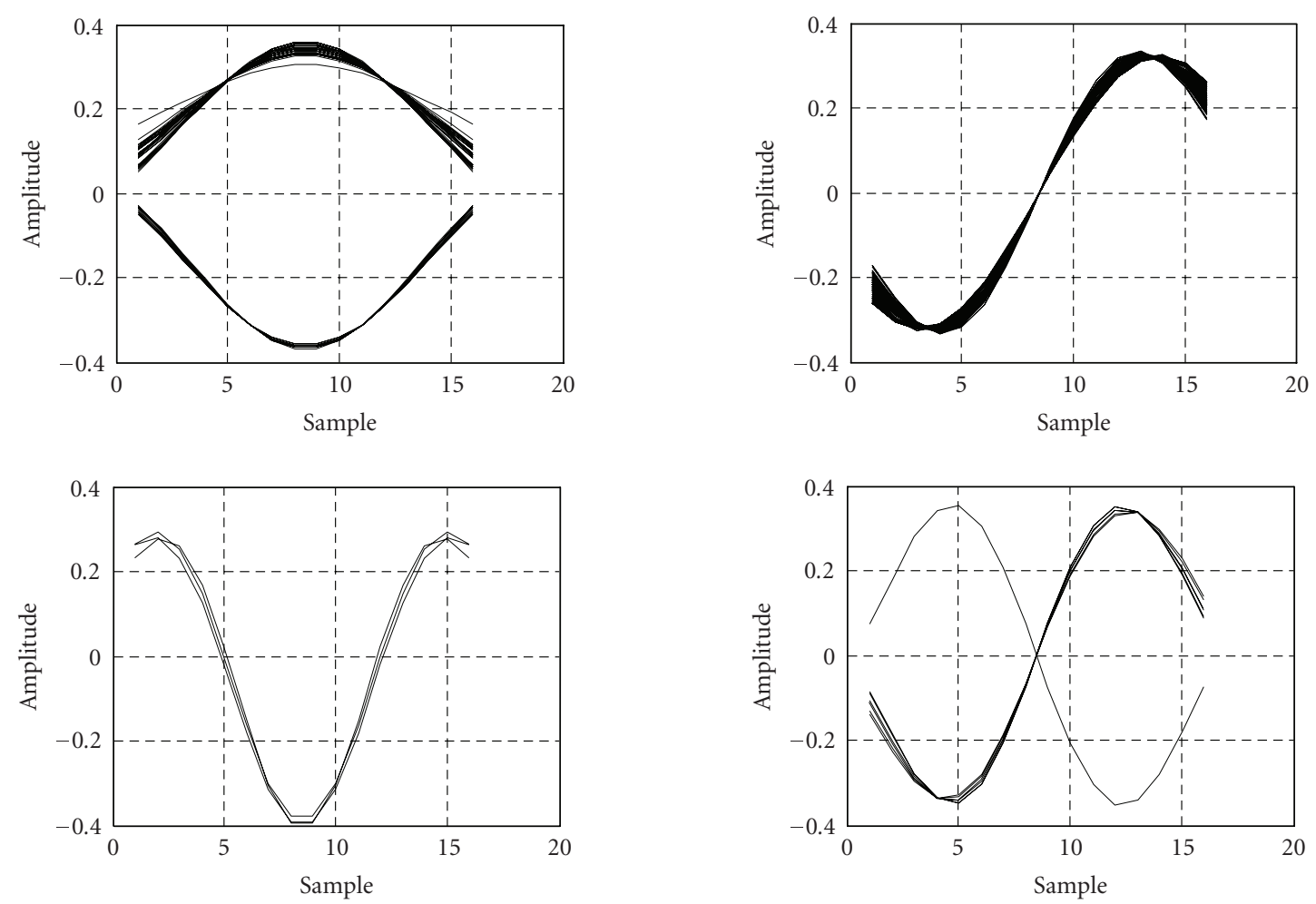

FIgURE 1: Some selected eigenvectors which exhibit similar patterns for $L_{F}=16$.

where

$$
r_{i}(d+1)=\frac{1}{L_{F}} \sum_{j=\left[(i-1) \cdot L_{F}+1\right]}^{\left[i \cdot L_{F}-d\right]} x_{j} x_{j+d}, \quad d=0,1,2, \ldots, L_{F}-1 .
$$

It should be noted that $R_{i}$ is a positive semidefinite, real symmetrical, and toeplitz matrix. The above-mentioned LMS process results in the following eigenvalue problem:

$$
R_{i} V_{k i}=\lambda_{k i} V_{k i}, \quad k=1,2, \ldots, L_{F}
$$

Obviously, $\lambda_{k i}$ and $V_{k i}$ are the eigenvalues and the eigenvectors of the problem under consideration. It is well known that the eigenvalues of the $R_{i}$ are also real and nonnegative. Moreover, the eigenvectors $V_{k i}$ are all orthonormal.

In (5), $V_{k i}$ can be streamed in accordance with the descending order of eigenvalues such that $\left(\lambda_{1 i} \geq \lambda_{2 i} \geq \cdots \geq\right.$ $\left.\lambda_{L_{F} i}\right)$. In this case in (5), the first eigenvector $V_{1 i}$ that has the highest energy associated with highest eigenvalue $\lambda_{1 i}$ represents the directions of greatest variations of the signal. Therefore, $V_{1 i}$ is called the major signature vector. In this regard, it may be suitable to approximate (5) with only first term as

$$
X_{i} \cong C_{1} V_{1 i}
$$

In this case, the frame length $L_{F}$ must be selected in such a way that almost maximum energy of $X_{i}$ is captured in (9).

The approximation $(\cong$ ) given by $(9)$ can be converted to an equality $(=)$ by means of an "envelope diagonal matrix
$A_{i}$ " for each frame. Thus, $X_{i}$ is computed as

$$
X_{i}=C_{i} A_{i} V_{1 i}
$$

In (10), diagonal entrees $a_{i r}$ of the matrix $A_{i}$ are determined in terms of the entrees $v_{1 i r}$ of the major signature vector $V_{1 i}$ and the samples of the original ECG signal $x_{i r}$ of the frame vector $X_{i}$ by

$$
a_{i r}=\frac{x_{i r}}{C_{i} v_{1 i r}}, \quad r=1,2, \ldots, L_{F}
$$

In this research work, many ECG signals were examined and thousands of frames were analyzed. It has been observed that patterns obtained by plotting $a_{i}(n)=\left(a_{i r}\right.$ versus frame in$\left.\operatorname{dex}-n=1,2, \ldots, L_{F}\right)$ and $v_{i}(n)=\left(v_{1 i r}\right.$ versus frame in$\left.\operatorname{dex}-n=1,2, \ldots, L_{F}\right)$ exhibit repetitive similarities. Some of these patterns are shown in Figures 1 and 2 .

Therefore, these similar patterns can be eliminated by comparison. It was experienced that use of Pearson's correlation coefficient which is defined by

$$
=\frac{\rho_{W Y}}{\sqrt{\left(\sum_{i=1}^{L} w_{i}^{2}-\left(\sum_{i=1}^{L} w_{i}\right)^{2} / L\right) \cdot\left(\sum_{i=1}^{L} y_{i}^{2}-\left(\sum_{i=1}^{L} y_{i}\right)^{2} / L\right)}}
$$

yields satisfactory reduction in the elimination process. In (12), $W=\left[\begin{array}{llll}w_{1} & w_{2} & \cdots & w_{L}\end{array}\right], Y=\left[\begin{array}{llll}y_{1} & y_{2} & \cdots & y_{L}\end{array}\right]$ designate two vectors which are subject to comparison. 

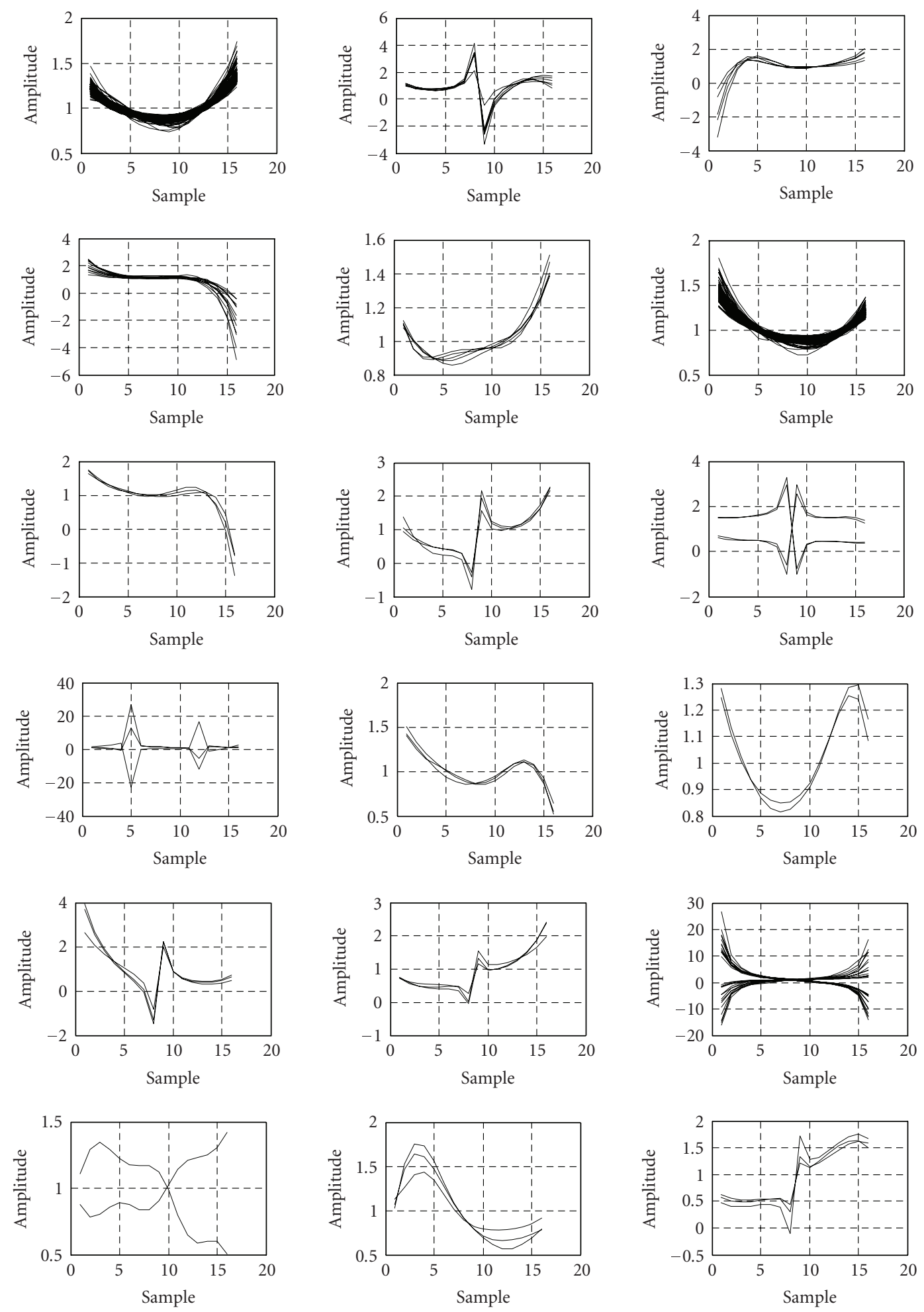

FIgURE 2: Some selected envelope vectors which exhibit similar patterns for $L_{F}=16$.

In this work, it is assumed that the two vectors are almost identical for $0.9 \leq \rho_{W Y} \leq 1$. Hence, similar patterns of signature and envelope vectors are eliminated accordingly.

In this work, once and for all, two types of sets were created by using reduced envelope and signature sequences. Re- duced signature vectors are collected under the predefined signature set (PSS) as $\left\{\varphi_{n_{s}}(n) ; n_{s}=1,2, \ldots, N_{S}\right\}$. Similarly, reduced envelope sequences or diagonal matrices are collected in the predefined envelope set (PES) as $\left\{\alpha_{n_{e}}(n) ; n_{e}=\right.$ $\left.1,2, \ldots, N_{E}\right\}$. In order to provide vision to the reader, some 

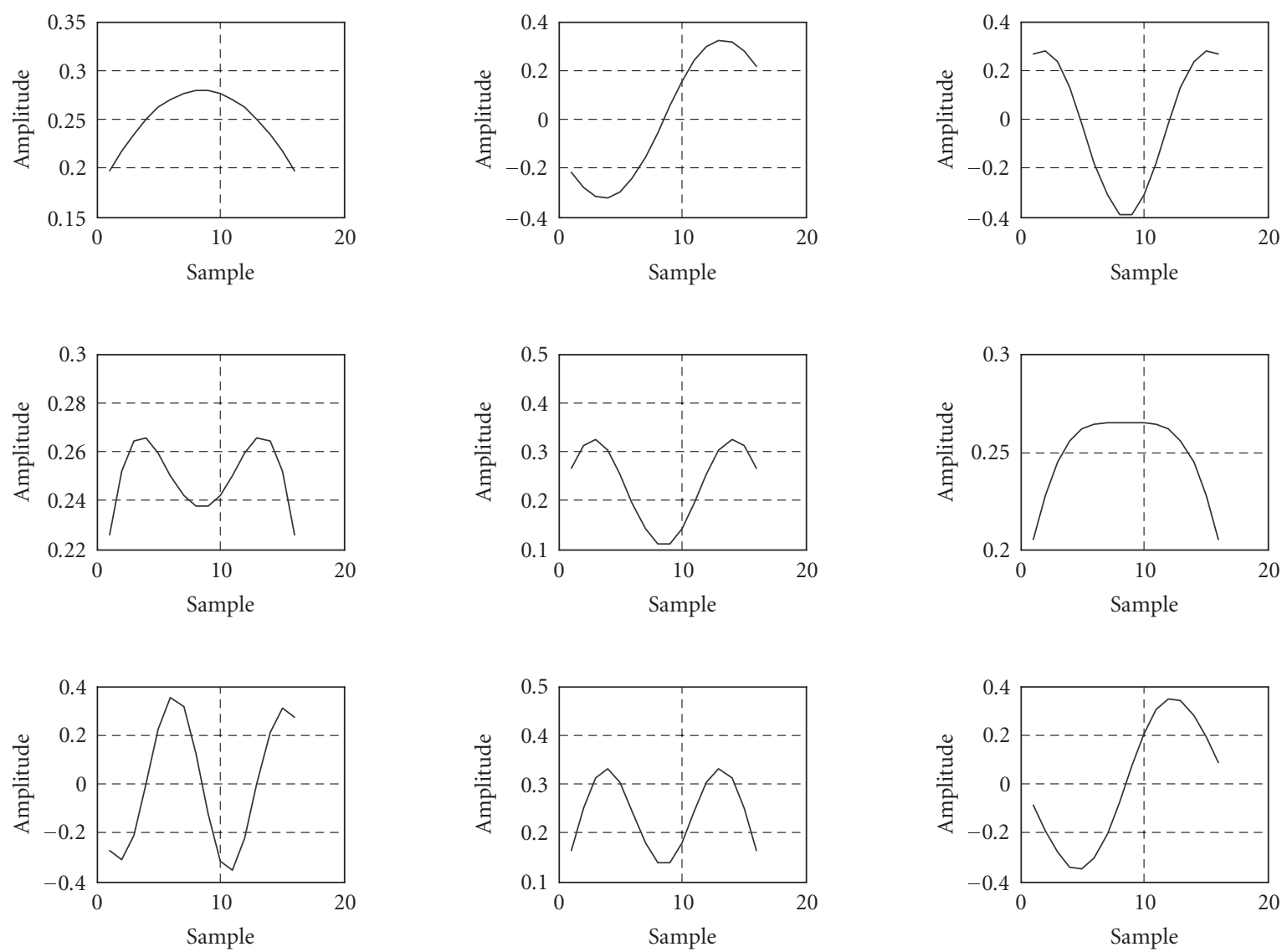

Figure 3: Some selected unique signature vectors in the predefined signature set for $L_{F}=16$.

selected unique signature and envelope vectors are shown in Figures 3 and 4 . Hence, any ECG signal frame $X_{i}$ can be represented in terms of the multiplication of predefined envelope $\alpha_{K}$ and signature $\varphi_{R}$ vectors pulled from PSS and PES with a constant $C_{i}$ in the least mean-square sense,

$$
X_{i} \cong C_{i} \alpha_{K} \varphi_{R} .
$$

In the following, first an algorithm is presented to generate predefined signature and envelope sets, which is essential for the reconstruction process of the measured ECG signals. Then, the reconstruction algorithm is introduced.

\section{ALGORITHMS}

The major philosophy of the proposed method to model ECG signals is based on the generation of the PSS and PES. Therefore, in this section first an algorithm is outlined to generate PSS and PES (Algorithm 1) then, reconstruction process of the ECG signals is detailed in Algorithm 2.

In this work, different values of $L_{F}$ (such as $L_{F}=8,16,20$, $24,32,48,64$ ) were selected to investigate the effect of the frame length on the quality of the reconstructed ECG signal by means of the PRD level. Details of this effort are given in the subsequent section.
Once PSS and PES are generated, then any ECG signal can be reconstructed frame by frame $\left(X_{i} \cong C_{i} \alpha_{K} \varphi_{R}\right)$ as implied by the main statement. It can be clearly seen that in this approach, the frame $i$ is reconstructed with three major quantities namely, the gain factor $C_{i}$, the index $R$ of the predefined signature vector $\varphi_{R}$ pulled from PSS, and the index $K$ of the predefined envelope sequence $\alpha_{K}$ pulled from PES. $\alpha_{K}$ and $\varphi_{R}$ are determined to minimize the LMS error which is described by means of the difference between the original frame $X_{i}$ and its model $X_{A i}=C_{i} E_{K} S_{R}$.

Details of the reconstruction process are given in Algorithm 2.

In the following section, simulation results of the new ECG modeling method are presented.

\section{SIMULATION RESULTS}

The proposed algorithms presented in the previous section were developed on a Mobil AMD Athlon 1.66 GHz processor. Predefined signature (PSS) and envelope sets (PES) were generated employing the digital ECG recordings of MIT arrhythmia database [19]. Using these PSS and PES, ECG signals of ECGMAN database [20] were reconstructed. Eventually, 

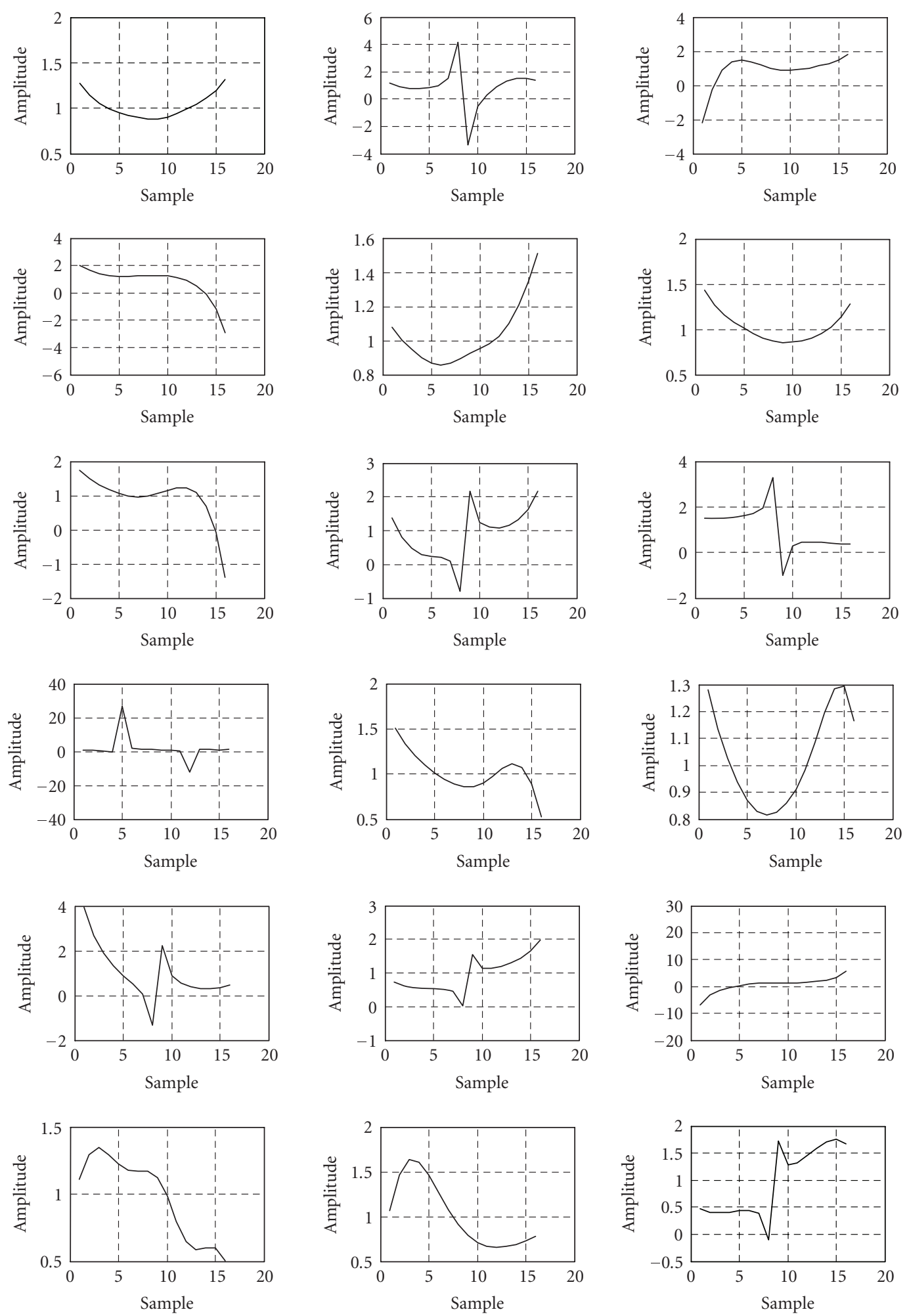

FIGURE 4: Some selected unique envelope vector in the predefined envelope set for $L_{F}=16$.

quality of the reconstructed signals was compared with those of classical methods. In the evaluation process of the proposed technique, compression ratios (CR) and percent root mean square differences (PRD) between the original and the reconstructed signals were computed $[21,22]$. In this regard,
$\mathrm{PRD}$ is defined by

$$
\mathrm{PRD}=\sqrt{\frac{\sum_{n=1}^{N}\left(x_{\mathrm{org}}(n)-x_{\mathrm{rec}}(n)\right)^{2}}{\sum_{n=1}^{N} x_{\mathrm{org}}^{2}(n)}} \times 100,
$$




\section{INPUTS}

(i) Main frame vector of the ECG signal $\{X(n), n=1,2, \ldots, N\}$.

(ii) $L_{F}$ : total number of samples in each frame under consideration.

\section{COMPUTATIONAL STEPS}

Step 1. Compute the total number of frames $N_{F}=N / L_{F}$.

Step 2. Divide $X(n)$ into frames $X_{i}$. In this case, the original ECG signal is represented by the main frame vector.

Step 3. For each frame $X_{i}$, compute the correlation matrix $R_{i}$.

Step 4. For each $R_{i}$, compute the eigenvalues $\lambda_{k i}$ in descending order with the corresponding eigenvectors.

Step 5. (a) Store the eigenvector which is associated with the maximum eigenvalue; call it the "signature vector" with the frame index $i$; and designate it as $V_{1 i}$.

(b) Compute the frame-scaling coefficient $C_{1}$ in the LMS sense to approximate $X_{i} \cong C_{1} V_{1 i}$.

Step 6. Repeat Steps 5(a) and 5(b) for all the frames $\left(i=1,2, \ldots, N_{F}\right)$. At the end of this loop, eigenvectors, which have maximum energy for each frame, will be collected.

Step 7. Compare all the collected eigenvectors obtained in Step 6 with an efficient algorithm. In this regard, Pearsons' correlation formula may be employed. Then, eliminate the ones which exhibit similar patterns. Thus, generate the predefined signature set PSS $=\left\{\varphi_{n_{s}}(n) ; n_{s}=1,2, \ldots\right.$, $\left.N_{S}\right\}$ with reduced number of eigenvectors $V_{1 i}$. Here, $N_{S}$ designates the total number of ones of kind signature patterns after the elimination.

Step 8. Compute the diagonal envelope matrix for each $C_{1 i} V_{1 i}$.

Step 9. Eliminate the envelope sequences which exhibit similar patterns with an efficient algorithm as in Step 7, and construct the predefined envelope set PES $=\left\{\alpha_{n_{e}}(n) ; n_{e}=1,2, \ldots, N_{E}\right\}$; Here, $N_{E}$ denotes the total number of ones of kind unique envelope patterns.

Algorithm 1: Generation of the predefined signature and envelope sets.

where $x_{\mathrm{org}}(n), x_{\text {rec }}(n)$, and $N$ designate original signal, reconstructed signal, and length of the original signal, respectively. The compression ratio (CR) is computed as follows:

$$
\mathrm{CR}=\frac{b_{\text {org }}}{b_{\text {rec }}} \quad \text { or } \quad \mathrm{CR}(\%)=\frac{b_{\text {org }}-b_{\text {rec }}}{b_{\text {org }}} \times 100 \text {, }
$$

where $b_{\text {org }}$ and $b_{\text {rec }}$ designate the total number of bits required representing original and reconstructed signals, respectively.

The MIT-BIH database contains 48 ECG recordings which are sampled at $360 \mathrm{~Hz}$ using a resolution of 12 bits/sample. On the other hand, the ECGMAN database includes 16 different digital ECG recordings with sampling rate of $500 \mathrm{~Hz}$ and $12 \mathrm{bits} / \mathrm{sample}$ resolution. In order to make fair comparison, MIT-BIH recordings were resampled at $500 \mathrm{~Hz}$ and all of the ECG recordings were normalized between -1 and +1 values.

In the generation of PSS and PES, Pearsons' correlation coefficient $\rho$ was varied over the interval $0.9 \leq \rho \leq 0.995$ for the elimination process. In the course of computations, effect of various frame lengths $L_{F}$ was investigated. Obviously, for each frame length $L_{F}$, one has to determine the total number of predefined signature and envelope sets, namely, $N_{S}$ and $N_{E}$ which in turn yield the total number of bits $b_{\text {Total }}=b_{C_{i}}+b_{R}+b_{K}$ required to represent the reconstructed ECG signals. In this presentation, $b_{C_{i}}, b_{R}$, and $b_{K}$ designate
TABLE 1: Variation of total number of bits with respect to frame length.

\begin{tabular}{rrrr}
\hline$L_{F}$ & $N_{S}$ & \multicolumn{1}{c}{$N_{E}$} & \multicolumn{1}{c}{$b_{\text {Total }}$} \\
\hline 8 & 15 & 512 & $6+4+9=19$ \\
16 & 16 & 1024 & $6+4+10=20$ \\
20 & 32 & 3836 & $6+5+12=23$ \\
24 & 125 & 7740 & $6+7+13=26$ \\
32 & 250 & 14378 & $6+8+14=28$ \\
48 & 779 & 30395 & $6+10+15=31$ \\
64 & 1736 & 58486 & $6+11+16=33$ \\
\hline
\end{tabular}

the least number of bits required to represent frame scaling coefficient $C_{i}$, and the integers $N_{S}$ and $N_{E}$, respectively. Comparative results are summarized in Table 1. It should be noted that for this research work, $b_{C_{i}}=6$ bits were good enough to code the entire frame-scaling coefficient $C_{i}$. For example, one second of original ECG recording contains 500 samples which in turn yield total number of $500 \times 12=6000 \mathrm{bits} / \mathrm{s}$. Employing the new method, if the frame length $L_{F}=8$ is chosen, then one second of ECG recording includes about $500 / 8=62.5$ frames. As it is seen from Table 1, choosing $\rho=0.995$, Algorithm 1 results in $N_{S}=15$ different signatures and $N_{E}=512$ different envelope patterns. Representing $N_{S}$ and $N_{E}$ with $b_{S}=4$ and $b_{E}=9$ bits, respectively, 


\section{INPUTS}

(i) ECG signal $\{X(n), n=1,2, \ldots, N\}$ to be modeled.

(ii) $L_{F}$ : number of samples in each frame.

(iii) $N_{S}$ and $N_{E}$; total number of the set elements in PSS and in PES, respectively. These integers are determined by Steps 7 and 9 of Algorithm 1, respectively.

(iv) The predefined signature set PSS $=\left\{\varphi_{R} ; R=1,2, \ldots, N_{S}\right\}$ created utilizing Algorithm 1 .

(v) The predefined envelope set PES $=\left\{\alpha_{K} ; K=1,2, \ldots, N_{E}\right\}$ created utilizing Algorithm 1.

\section{COMPUTATIONAL STEPS}

Step 1. Divide $X$ into frames $X_{i}$ of length $L_{F}$ as in Algorithm 1. In this case, the original ECG signal is represented by the main frame Vector.

Step 2. (a) For each frame $i$, pull an appropriate signature vector $\varphi_{R}$ from PSS such that the distance or the total error $\delta_{\widetilde{R}}=\left\|X_{i}-C_{\widetilde{R}} \varphi_{\widetilde{R}}\right\|^{2}$ is minimum for all $\widetilde{R}=1,2, \ldots, R, \ldots, N_{S}$. This step yields the index $R$ of the $\varphi_{R}$. In this case, $\delta_{\widetilde{R}}=\min \left\|X_{i}-C_{\widetilde{R}} \varphi_{\widetilde{R}}\right\|^{2}=\left\|X_{i}-C_{R} \varphi_{R}\right\|^{2}$.

(b) Store the index number $R$ that refers to $\varphi_{R}$, in this case, $X_{i} \approx C_{R} \varphi_{R}$.

Step 3. (a) Pull an appropriate envelope sequence (or diagonal envelope matrix) $\alpha_{K}$ from PES such that the error is further minimized for all $\tilde{K}=1,2, \ldots, K, \ldots, N_{E}$. Thus, $\delta_{K}=\min \left\{\left\|X_{i}-C_{R} \alpha_{\widetilde{K}} \varphi_{R}\right\|^{2}\right\}=$ $\left\|X_{i}-C_{R} \alpha_{K} \varphi_{R}\right\|^{2}$. This step yields the index $K$ of the $\alpha_{K}$.

(b) Store the index number $K$ that refers to $\alpha_{K}$. It should be noted that at the end of this step, the best signature vector $\varphi_{R}$ and the best envelope sequence $\alpha_{K}$ are found by appropriate selections. Hence, the frame $X_{i}$ is best described in terms of the patterns of $\alpha_{K}$ and $\varphi_{R}$, that is, $X_{i} \approx C_{R} \alpha_{K} \varphi_{R}$.

Step 4. Having fixed $\varphi_{R}$ and $\alpha_{K}$, one can replace $C_{R}$ by computing a new gain factor $C_{i}=\left(\alpha_{K} \varphi_{R}\right)^{T} X_{i} /$ $\left(\alpha_{K} \varphi_{R}\right)^{T}\left(\alpha_{K} \varphi_{R}\right)$ to further minimize the distance between the vectors $X_{i}$ and $C_{R} \alpha_{K} \varphi_{R}$ in the LMS sense. In this case, the global minimum of the error is obtained and it is given by $\delta_{\mathrm{Global}}=\| X_{i}-$ $C_{R} \alpha_{K} \varphi_{R} \|^{2}$. At this step, the frame sequence is approximated by $X_{A i}=C_{R} \alpha_{K} \varphi_{R}$.

Step 5. Repeat the above steps for each frame to reconstruct the ECG signal.

Algorithm 2: Reconstruction of ECG signals by using PSEVS.

TABLE 2: CR and average PRD associated with PSEVS for different frame lengths.

\begin{tabular}{rrrc}
\hline$L_{F}$ & CR & CR $(\%)$ & Average PRD (\%) \\
\hline 8 & 5.05 & 80.2 & 2.250 \\
16 & 9.60 & 89.58 & 3.504 \\
20 & 10.44 & 90.42 & 4.546 \\
24 & 11.08 & 91.00 & 5.334 \\
32 & 13.72 & 92.71 & 5.939 \\
48 & 18.58 & 94.62 & 8.033 \\
64 & 23.28 & 95.71 & 9.359 \\
\hline
\end{tabular}

total number of bits required to represent one ECG frame is $b_{\text {Total }}=6+4+9=19$ bits which in turn yield a compression ratio of $\mathrm{CR}=8 \times 12 / 19=5.05$. On the other hand, if $L_{F}=64$ is selected, then Algorithm 1 reveals that $N_{S}=1736$ and $N_{E}=58486$ which corresponds to 33 bits per frame representation. In this case, compression ratio is $\mathrm{CR}=64 \times 12 / 33=23.28$.

Once PSS and PES were generated, then 16 ECG signals given by ECGMAN database were reconstructed for quality assessment of the proposed technique. Table 2 illustrates the coding performance and the average PRD of the proposed method in terms of CR, CR(\%), and PRD for different frame lengths.
As it can be seen from Tables 1 and 2, the proposed method exhibits relative compression ratios in the range of $80.2 \%-95.71 \%$ with average PRD varying between $2.250 \%$ and $9.359 \%$.

It should be noted that in the existing literature, acceptable values of PRD are reported as less than 10\% [21]. Thus, Table 2 indicates that the proposed method results in high compression ratio with very good PRD levels. In Figure 5, various assessment means are given to evaluate the proposed method.

For the sake of visual inspection, for the frame length $L_{F}=16$ cases, various original ECG signals selected from ECGMAN database and the signals constructed via new method are depicted in Figure 6.

Figure 7 shows the PRD variations of the reconstructed signals given in Figure 6. As it is seen from Figure 7 that average PRD level is about 3.5\% which corresponds to a high quality of reconstruction with high compression rate of 9.6. On the other hand, the average reconstruction time of the proposed method is approximately 1 second.

In order to carry out fair evaluations among the existing and our newly proposed techniques, first the conventional methods of [2-16] were programmed in our laboratory, then using the same ECG signals given by the MIT-BIH arrhythmia and ECGMAN database, these methods and proposed method were evaluated. Eventually, compression ratios and 


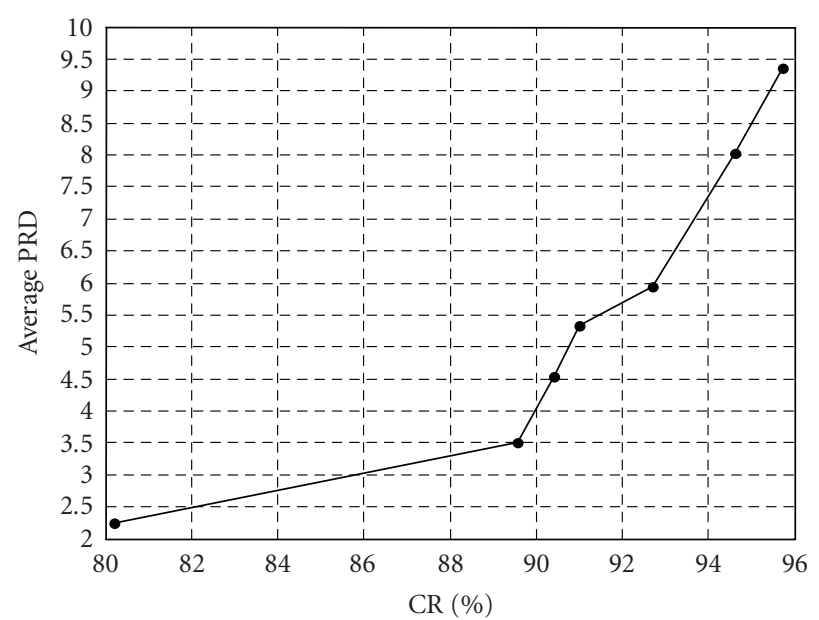

(a)

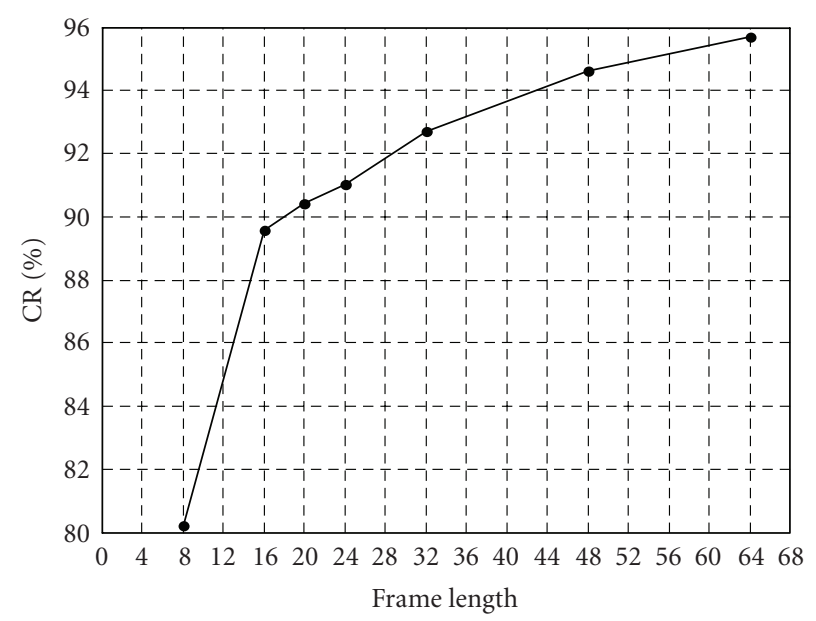

(b)

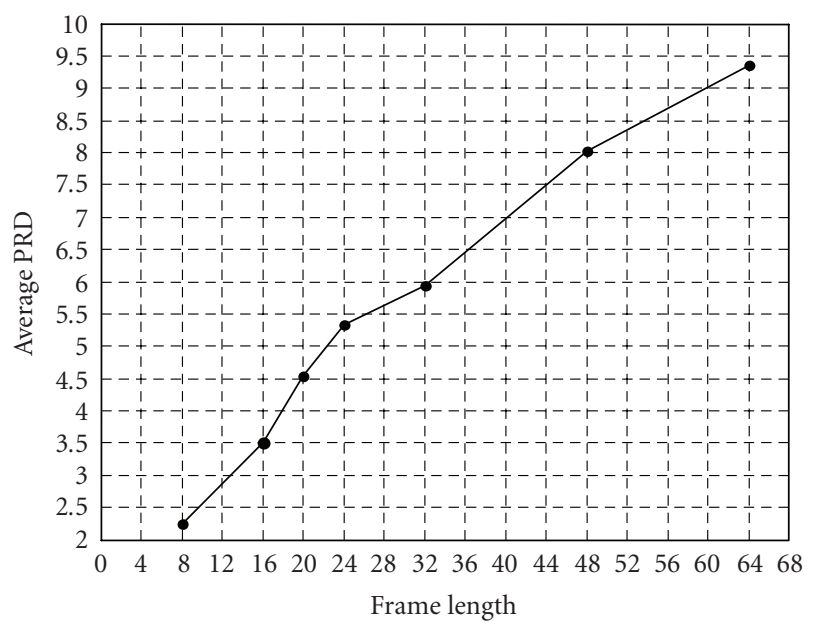

(c)

Figure 5: (a) Performance assessment of the new technique: variation of average PRD with respect to CR(\%). (b) Performance assessment of the new technique: variation of $\mathrm{CR}(\%)$ with respect to frame length. (c) Performance assessment of the new technique: variation of average PRD with respect to frame length.
TABLE 3: Comparison of the proposed method with various ECG compression methods.

\begin{tabular}{lrc}
\hline Method & CR & PRD (\%) \\
\hline TP [2] & 2.0 & 5.3 \\
AZTEC [2] & 10.0 & 28.1 \\
CORTES [2] & 4.8 & 7.0 \\
FAN/SAPA [2] & 3.0 & 4.0 \\
MSAPA/CSAPA [3] & 5.0 & 3.5 \\
SAIES [4] & 5.9 & 16.3 \\
Vector quantization of wavelet coefficients [3] & 10.0 & 5.5 \\
Classified vector quantization [3] & 8.6 & 24.5 \\
Peak peaking (spline) with entropy encoding [3] & 10.0 & 14.0 \\
ADPCM [9] & 6.0 & 6.9 \\
& 9.6 & 4.09 \\
Mean-shape vector quantizer [3] & 10.5 & 5.26 \\
& 23.2 & 9.82 \\
Predefined signature & 9.6 & 3.504 \\
and envelope vector & 10.44 & 4.546 \\
Sets (proposed & 23.28 & 9.359 \\
\hline
\end{tabular}

the percent root mean-squared errors were compared. Thus, the comparison results in Table 3 are obtained.

These results indicate that the proposed method provides a higher compression ratio with lower PRD values over all the existing lossy compression methods.

\section{CONCLUSIONS AND DISCUSSION}

In this paper, a new method to represent ECG signals is presented. The proposed technique is based on the generation of the predefined signature and envelope vector sets. In the proposed technique, each frame of any ECG signal is described by multiplying three major quantities, namely frame-scaling coefficient $C_{i}$, the frame signature vector $\varphi_{R}$, and diagonal envelope matrix $\alpha_{K}$. Signature and envelope patterns are selected from the corresponding predefined signature and envelope vector sets that are formed by using MIT-BIH arrhythmia database which contains much cardiac pathology. In the reconstruction process, each ECG frame is fully identified with the frame-scaling coefficient $C_{i}$ and the indices $R$ and $K$ of the predefined signature and the envelope patterns, respectively.

The selection of the appropriate database is very important in order to construct the PSV and PEV sets. The selected database must contain a large set of ECG beats and many examples of much cardiac pathology. Therefore, in this work, the MIT-BIH arrhythmia database which has the desired properties was used in order to construct the PSV and PEV sets. On the contrary, ECGMAN database which contains 16 ECG signals is a small database. If ECGMAN database was used for constructing the PSV and PEV sets, the 

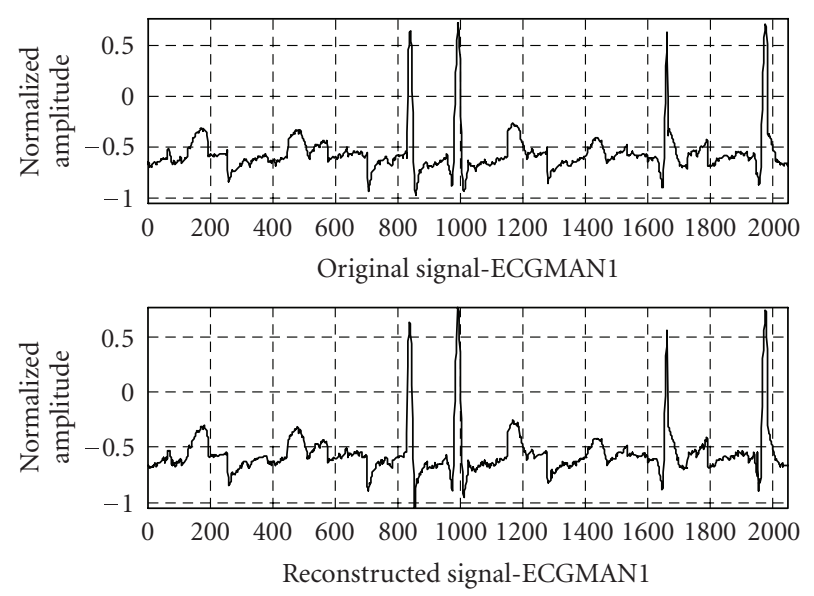

(a) $\mathrm{PRD}=3.2473, \mathrm{CR}=9.6$, reconstruction time: 1.061 seconds.
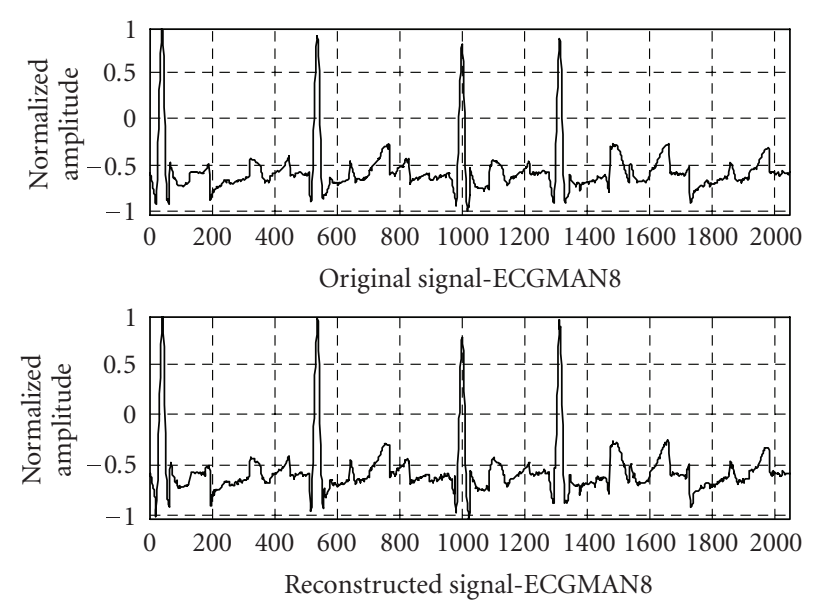

(c) $\mathrm{PRD}=3.2954, \mathrm{CR}=9.6$, reconstruction time: 1.072 seconds.
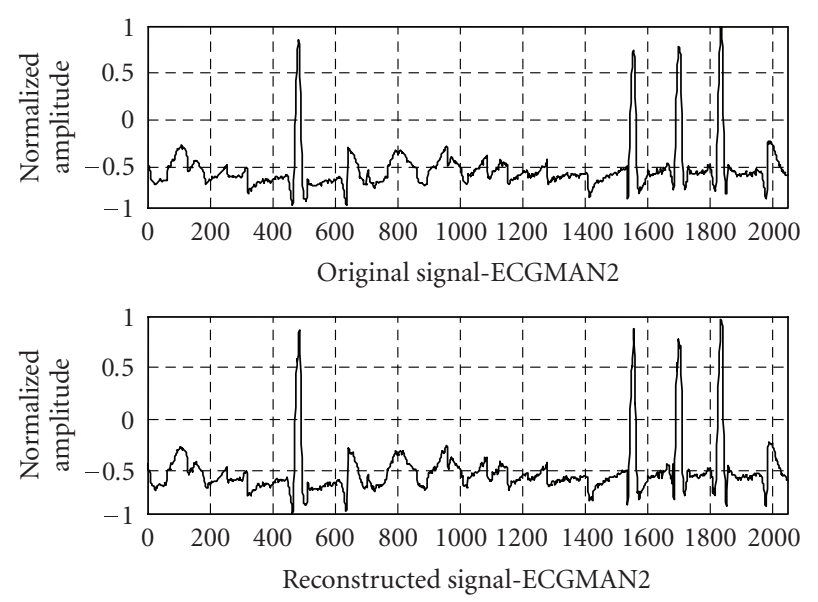

(b) $\mathrm{PRD}=4.1351, \mathrm{CR}=9.6$, reconstruction time: 1.072 seconds.
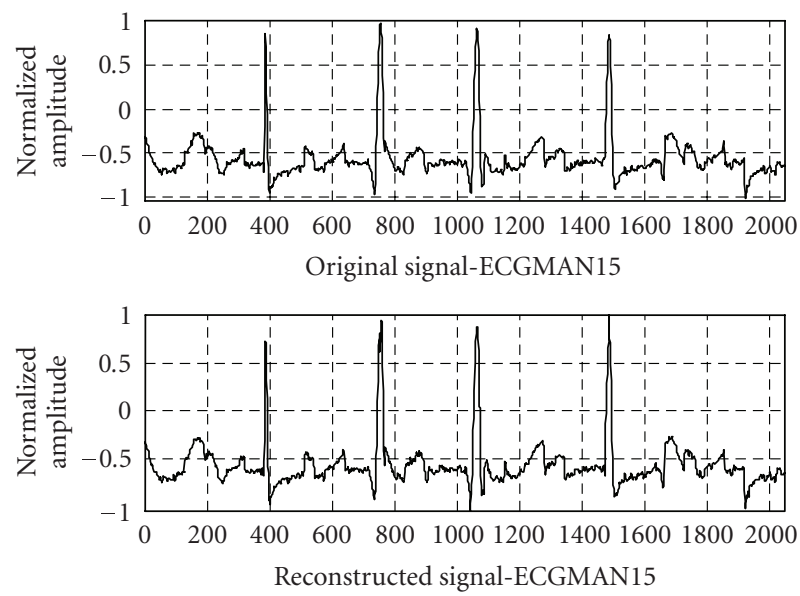

(d) $\mathrm{PRD}=3.7808, \mathrm{CR}=9.6$, reconstruction time: 0.952 seconds.

FIgURE 6: Original and reconstructed ECG signals for $L_{F}=16$.

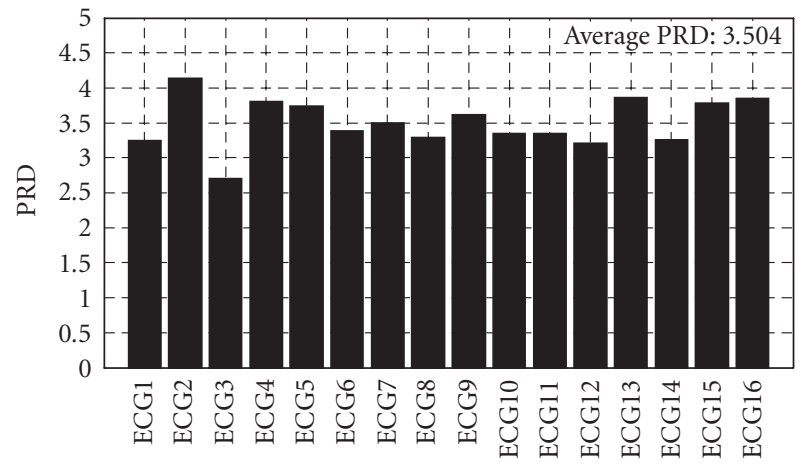

ECG signals

FIGURE 7: PRD values associated with ECG signals in the ECGMAN database for $L_{F}=16$.

obtained performance would be poor. Hereby, the ECGMAN database could not be used to construct the PSV and PEV sets.
It should be noted that if the ECG signals in the MIT-BIH arrhythmia database were used for the test signal, the performance of our method would be better than the reported results in our paper. Because the ECG signal which is used to test is the same as the ECG signal which is used for constructing the PSV and PEV sets. In order to avoid this situation, two different databases were used to construct these sets and test the performance of our method.

It was briefly explained above; our training database is MIT-BIH database which includes 48 different ECG signals. The test set was selected from the different domains which are unlike the training data set. As a result, it can be concluded that our method is not a lead-specific method.

While preserving the diagnostic information, the proposed method provides significant data compression rate with low PRD values over the other available methods given in the current literature. The main superiority of the proposed method is that it does not need to employ any QRS detection algorithm. Thus, it requires less computation time. 


\section{ACKNOWLEDGMENTS}

The present work was supported by the Research Fund of Istanbul University, Project no. 400/03062005, and UDP440/10032005.

\section{REFERENCES}

[1] D. L. Hudson and M. E. Cohen, "Intelligent analysis of biosignals," in Proceedings of the 27th Annual International Conference of the Engineering in Medicine and Biology Society (EMBS '05), pp. 323-326, Shanghai, China, September 2005.

[2] S. M. S. Jalaleddine, C. G. Hutchens, R. D. Strattan, and W. A. Coberly, "ECG data compression techniques - a unified approach," IEEE Transactions on Biomedical Engineering, vol. 37, no. 4, pp. 329-343, 1990.

[3] J. L. Cârdenas-Barrera and J. V. Lorenzo-Ginori, "Mean-shape vector quantizer for ECG signal compression," IEEE Transactions on Biomedical Engineering, vol. 46, no. 1, pp. 62-70, 1999.

[4] S. M. S. Jalaleddine and C. G. Hutchens, "SAIES - a new ECG data compression algorithm," Journal of Clinical Engineering, vol. 15, no. 1, pp. 45-51, 1990.

[5] S.-G. Miaou and J.-H. Larn, "Adaptive vector quantisation for electrocardiogram signal compression using overlapped and linearly shifted codevectors," Medical and Biological Engineering and Computing, vol. 38, no. 5, pp. 547-552, 2000.

[6] C.-C. Sun and S.-C. Tai, "Beat-based ECG compression using gain-shape vector quantization," IEEE Transactions on Biomedical Engineering, vol. 52, no. 11, pp. 1882-1888, 2005.

[7] K. Skretting, K. Engan, and J. H. Husøy, "ECG compression using signal dependent frames and matching pursuit," in Proceedings of IEEE International Conference on Acoustics, Speech and Signal Processing (ICASSP '05), vol. 4, pp. 585-588, Philadelphia, Pa, USA, March 2005.

[8] E. B. de Lima Filho, E. A. B. da Silva, M. B. de Carvalho, W. S. da Silva Júnior, and J. Koiller, "Electrocardiographic signal compression using multiscale recurrent patterns," IEEE Transactions on Circuits and Systems I: Regular Papers, vol. 52, no. 12, pp. 2739-2753, 2005.

[9] H. Lee and K. M. Buckley, "ECG data compression using cut and align beats approach and 2-D transforms," IEEE Transactions on Biomedical Engineering, vol. 46, no. 5, pp. 556-564, 1999.

[10] J.-J. Wei, C.-J. Chang, N.-K. Chou, and G.-J. Jan, "ECG data compression using truncated singular value decomposition," IEEE Transactions on Information Technology in Biomedicine, vol. 5, no. 4, pp. 290-299, 2001.

[11] S.-G. Miaou, H.-L. Yen, and C.-L. Lin, "Wavelet-based ECG compression using dynamic vector quantization with tree codevectors in single codebook," IEEE Transactions on Biomedical Engineering, vol. 49, no. 7, pp. 671-680, 2002.

[12] H.-H. Chou, Y.-J. Chen, Y.-C. Shiau, and T.-S. Kuo, "An effective and efficient compression algorithm for ECG signals with irregular periods," IEEE Transactions on Biomedical Engineering, vol. 53, no. 6, pp. 1198-1205, 2006.

[13] S.-C. Tai, C.-C. Sun, and W.-C. Yan, "A 2-D ECG compression method based on wavelet transform and modified SPIHT," IEEE Transactions on Biomedical Engineering, vol. 52, no. 6, pp. 999-1008, 2005.

[14] S.-G. Miaou and S.-N. Chao, "Wavelet-based lossy-to-lossless ECG compression in a unified vector quantization framework," IEEE Transactions on Biomedical Engineering, vol. 52, no. 3, pp. 539-543, 2005.
[15] B. S. Kim, S. K. Yoo, and M. H. Lee, "Wavelet-based lowdelay ECG compression algorithm for continuous ECG transmission," IEEE Transactions on Information Technology in Biomedicine, vol. 10, no. 1, pp. 77-83, 2006.

[16] Y. Zigel, A. Cohen, and A. Katz, "ECG signal compression using analysis by synthesis coding," IEEE Transactions on Biomedical Engineering, vol. 47, no. 10, pp. 1308-1316, 2000.

[17] Ü. Güz, H. Gürkan, and B. S. Yarman, "A novel method to represent the speech signals by using language and speaker independent predefined functions sets," in Proceedings of IEEE International Symposium on Circuits and Systems (ISCAS '04), vol. 3, pp. 457-460, Vancouver, BC, Canada, May 2004.

[18] B. S. Yarman, Ü. Güz, and H. Gürkan, "On the comparative results of "SYMPES: a new method of speech modeling"," $A E U$ - International Journal of Electronics and Communications, vol. 60, no. 6, pp. 421-427, 2006.

[19] G. B. Moody, The MIT-BIH Arrhythmia Database CD-ROM, Harvard-MIT Division of Health Sciences and Technology, Cambridge, Mass, USA, 2nd edition, 1992.

[20] A Karaş, Elektriksel İşaretlerin Temel Tanım Fonksiyonlarıyla Karakterizasyonu, Ph.D. thesis, Department of Electrical and Electronic Engineering, Institute of Science, Istanbul University, Istanbul, Turkey, 1997.

[21] Y. Zigel, A. Cohen, and A. Katz, "The weighted diagnostic distortion (WDD) measure for ECG signal compression," IEEE Transactions on Biomedical Engineering, vol. 47, no. 11, pp. 1422-1430, 2000.

[22] M. Blanco-Velasco, F. Cruz-Roldan, J. I. Godino-Llorente, J. Blanco-Velasco, C. Armiens-Aparicio, and F. López-Ferreras, "On the use of PRD and CR parameters for ECG compression," Medical Engineering and Physics, vol. 27, no. 9, pp. 798802, 2005.

Hakan Gürkan received the B.S., M.S., and Ph.D. degrees in electronics and communication engineering from the Istanbul Technical University (ITU), Istanbul, Turkey, in 1994, 1998, and 2005, respectively. He was a Research Assistant in the Department of Electronics Engineering, Engineering Faculty, Işık University, Istanbul, Turkey. He has been an Instructor in the Department of Electronics Engineering, Engineering Faculty, Işık University, Istanbul, Turkey, since 2005. His current interests are in digital signal processing, mainly with biomedical and speech signals modeling, representation, and compression.

Ümit Güz graduated from Istanbul Pertevniyal High School in 1988 and The Department of Computer Programming, Yıldız Technical University, Istanbul, Turkey, in 1990. He received the B.S. degree with high honors from the Department of Electronics Engineering, College of Engineering, Istanbul University, Turkey, in 1994. He received M.S. and Ph.D. degrees in electronics engineering with high honors

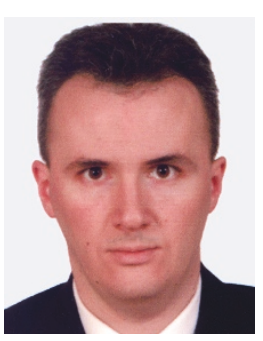
from the Institute of Science, Istanbul University in 1997 and 2002, respectively. From 1995 to 1998, he was a Research and Teaching Assistant in the Department of Electronics Engineering, Istanbul University. He has been a Faculty Member in the Department of Electronics Engineering, Engineering Faculty, Işık University, 
Istanbul, Turkey since 1998. He was awarded a Postdoctoral Research Fellowship by the Scientific and Technological Research Council of Turkey in 2006. He was accepted as an International Fellow by the Stanford Research Institute (SRI) International, Speech Technology and Research (STAR) Laboratory in 2006. He was awarded a J. William Fulbright Postdoctoral Research Fellowship in 2007. He was accepted as an International Fellow by the International Computer Science Institute (ICSI) Speech Group, University of California, Berkeley, in 2007. His research interest covers speech processing, modeling, coding, compression, automatic speech recognition, natural language processing, and biomedical signal processing.

B. Siddık Yarman received the B.S. degree in electrical engineering from Istanbul Technical University, Turkey (1974); M.E.E.E. degree from Electro-Math Stevens Institute of Technology Hoboken, NJ, 1977; $\mathrm{Ph} . \mathrm{D}$. degree in electrical engineering and mathematics from Cornell University, Ithaca, NY, 1981; Technical staff, Microwave Technology Centre, RCA David Sarnoff Re-

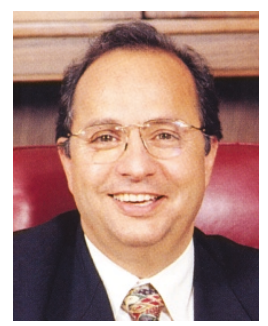
search Center, Princeton, NJ (1982-1984);

Professor, Alexander von Humboldt Fellow, Ruhr University, Bochum, Germany (1987-1994); Founding Director, STFA Defense Electronic Corporation, Turkey (1986-1996); Professor, Chair, Defense Electronics, Director, Technology and Science School, Istanbul University (1990-1996); Founding President of Işık University, Istanbul, Turkey (1996-2004); Chief Advisor to Prime Ministry Office, Turkey (1996-2000); Young Turkish Scientist Award (1986) and Technology Award (1987) of National Research Council of Turkey; International Man of the Year in Science and Technology, Cambridge Biography Center of UK (1998); Member of the Academy of Science of New York (1994); IEEE Fellow. Author of more than 100 papers, 4 US patents. Fields of interests include design of matching networks and microwave amplifiers, mathematical models for speech and biomedical signals. He has been back to Istanbul University since October 2004 and he is spending his sabbatical year of 2006-2007 at Tokyo Institute of Technology, Tokyo, Japan. 